\title{
Enquête : niveau de connaissance de la population au sujet du radon en Suisse
}

\author{
M. GRUSON ${ }^{1}$, C. MURITH ${ }^{1}$, S. RUMO ${ }^{2}$
}

(Manuscrit reçu le 26 août, accepté le 29 octobre 2009)

RÉSUMÉ Une enquête a été réalisée en 1995, afin d'appréhender le niveau de connaissance des Suisses sur le thème du radon. En 2008, une deuxième enquête, basée sur une méthodologie similaire, a été menée par l'OFSP. La nouvelle étude a montré qu'environ $40 \%$ de la population suisse a déjà entendu parler du radon, ce qui représente une augmentation de $8 \%$ par rapport à l'enquête de 1995 . La majorité des personnes interrogées savaient que le radon provoque le cancer du poumon, mais pensaient que le gaz induit aussi d'autres effets sur la santé, notamment la migraine et les affections dermatologiques. Par ailleurs, les personnes âgées, avec un niveau élevé de formation et propriétaires immobiliers connaissaient tendanciellement mieux la problématique du radon que le grand public. Les habitants des régions à risque élevé ont obtenus des résultats nettement meilleurs, ce qui démontre que les campagnes d'information dans ces régions ont porté leurs fruits. Par contre, un effort d'information supplémentaire est nécessaire dans les communes à risque léger et moyen, dans lesquelles la majeure partie de la population réside.

ABSTRACT Survey: Knowledge level of the population about radon in Switzerland.

In 1995, a survey was conducted in order to investigate levels of knowledge about radon among the Swiss population. In 2008, a second survey, using a similar methodology, was carried out by the FOPH. The new study showed that about $40 \%$ of the Swiss population has heard of radon, which represents an increase of $8 \%$ over the 1995 survey. Most of the respondents knew that radon causes lung cancer, but believed that the gas also produces other health effects - in particular, migraine and skin conditions. In addition, older people, those with a high level of education and property owners tended to be more familiar with the radon issue than the public at large. The inhabitants of high-risk regions achieved markedly better results, which demonstrate that information campaigns in these regions have been successful. At the same time, additional communication efforts are required in low- and mediumrisk municipalities, where the majority of the population lives.

Keywords: Radon / Switzerland / survey / population / communication

\section{Introduction}

Suite à l'introduction de l'ordonnance sur la radioprotection (ORaP, RS 814.501) en juin 1994 (ORaP, 1994), l'Office fédéral de la santé publique (OFSP) a été chargé de la gestion du programme suisse du radon, et notamment de l'information du public sur cette problématique. Les autorités cantonales sont, quant à elles, responsables de l'exécution du programme.

1 Office fédéral de la santé publique, Section risques radiologiques, 3003 Berne, Suisse.

2 CBC Marketing Research, Marktgasse 32, 3011 Berne, Suisse. 
En 1995, une enquête a été réalisée (IPSO, 1995), afin d'appréhender le niveau de connaissance des Suisses sur ce thème avant toute intervention des autorités cantonales. Cette étude a montré que $31,5 \%$ de la population avait déjà entendu parler du radon.

En 2008, une deuxième enquête, basée sur la même méthodologie que la première, a été menée par l'OFSP, en collaboration avec l'institut CBC Marketing Research à Berne. Le questionnaire constitué de quatre volets a été retravaillé et actualisé, car plus de 10 ans séparent les deux enquêtes.

Seules 4 questions de l'étude de 1995 ont été conservées (sans compter les questions sociodémographiques) et serviront d'indicateurs de l'évolution de la connaissance en Suisse :

- Avez-vous déjà entendu parler du radon ?

- Selon vous, le radon est-il un problème à l'intérieur ou à l'extérieur des bâtiments?

- À votre avis, le radon est-il nocif pour la santé ?

- Pensez-vous que des solutions permettent de réduire la concentration en radon dans un bâtiment?

L'étude a trois objectifs principaux :

- déterminer l'évolution de la connaissance du radon entre 1995 et 2008, à l'aide des quatre indicateurs ;

- appréhender le niveau de connaissance actuel de l'ensemble de la population sur le radon ;

- saisir ce niveau en fonction du risque en radon dans la commune de résidence.

\section{Méthodologie}

La méthodologie choisie est similaire à la première enquête, qui est une étude CATI (Computer Aided Telephone Interviewing), c'est-à-dire une interview téléphonique auprès des ménages résidant en Suisse. L'entretien téléphonique est la méthode la plus appropriée pour atteindre les ménages sur l'ensemble du territoire suisse à moindre coût et pendant une période déterminée. Les interviews, d'une durée moyenne de 11 minutes, ont été réalisées du 7 octobre au 20 novembre 2008 depuis la centrale téléphonique de CBC à Berne. Tous les enquêteurs ont reçu une instruction de départ avec au minimum un contrôle de qualité par enquêteur (écoute du superviseur) pendant l'étude.

\section{1. Échantillonage}

Les entretiens ont été réalisés auprès de 800 ménages privés, soit 100 entretiens en Suisse italienne, 200 en Suisse romande et 500 en Suisse allemande, ceci afin de garantir un échantillon suffisant, notamment dans la région italophone. 
Comme dans l'enquête de 1995 et afin d'obtenir un échantillon représentatif de la population résidente et de la structure sociodémographique de la population suisse (OFS, 2007), des quotas basés sur les données de l'Office fédéral de la statistique (OFS, 2006) ont été appliqués, à savoir :

- canton : il est important que des cantons fortement peuplés comme Zürich (107 entretiens réalisés sur 800) ou Berne (73 entretiens) aient plus de poids que des cantons comme le Jura (17 entretiens) ou les Grisons (20 entretiens) ;

- ville-campagne : le nombre d'entretiens par canton doit correspondre à la répartition de la population entre la ville et la campagne. Par exemple dans le canton de Zürich la quasi-totalité des entretiens a été réalisée en ville, soit 105 sur 107 ;

- genre : $49 \%$ d'hommes et $51 \%$ de femmes ;

- âge : 263 entretiens auprès des 18-34 ans, 329 entretiens auprès des 35-54 ans et 208 entretiens auprès des 55-74 ans.

TABLEAU I

Nombre d'interviews par canton.

Number of interviews per canton.

\begin{tabular}{lclc}
\hline Canton & Nombre & Canton & Nombre \\
\hline Argovie & 42 & Nidwald & 5 \\
Appenzell Rhodes-Intérieures & 0 & Obwald & 4 \\
\hline Appenzell Rhodes-Extérieures & 6 & St-Gall & 48 \\
Berne & 73 & Schaffhouse & 8 \\
Bâle-Campagne & 27 & Soleure & 24 \\
Bâle-Ville & 21 & Schwyz & 14 \\
Fribourg & 36 & Tessin & 100 \\
Genève & 49 & Thurgovie & 23 \\
Glaris & 4 & Uri & 3 \\
Grisons & 20 & Valais & 35 \\
Jura & 17 & Vaud & 65 \\
Lucerne & 31 & Zoug & 10 \\
Neuchâtel & 28 & Zürich & 107 \\
\hline
\end{tabular}

Cet échantillon permet de garantir un intervalle de confiance de $95 \%$ et une marge d'erreur de $\pm 3,46 \%$ généralement admis pour un sondage d'opinion (Kay et Cliffs, 2004).

Les numéros de téléphones ont été aléatoirement sélectionnés dans l'annuaire téléphonique, proportionnellement au nombre d'habitants par canton et au critère «ville-campagne ». Ensuite, le programme CATI contrôlait qu'il y ait suffisamment 
d'adresses pour atteindre les quotas fixés. Dans une deuxième étape, l'enquêteur sélectionnait des personnes correspondant aux quotas sociodémographiques prédéfinis (âge et genre).

Près de 32 adresses ont été utilisées pour une interview, car d'une part, environ $11 \%$ des personnes contactées étaient hors quotas et d'autre part, environ $23 \%$ n'ont pas souhaitées participer au sondage. Ce taux de refus est relativement bas, car il est généralement situé entre 20 et $40 \%$ pour ce type d'étude.

Les résultats finaux ont été pondérés afin de revenir à la structure de la population suisse en 2008 , qui a été calculée selon le nombre d'entrées dans l'annuaire téléphonique, en éliminant les entreprises et les doublons :

- Suisse italienne : $4,5 \%$;

- Suisse romande : $23,6 \%$;

- Suisse alémanique : $71,9 \%$.

\subsection{Stratification}

Plusieurs critères ont été utilisés pour la stratification de l'analyse :

- paramètres sociodémographiques,

- paramètres significatifs,

- groupe du collectif+.

\subsubsection{Paramètres sociodémographiques}

Les paramètres sociodémographiques sont les suivants :

- genre et âge : 18-34 ans, 35-54 ans et 55-74 ans ;

- région linguistique : régions alémanique, francophone et italophone;

- formation : contrairement au revenu, qui s'applique à toutes les personnes du foyer, le type de formation est un bon indicateur du niveau socioculturel de la personne interrogée, raison pour laquelle ce paramètre a été retenu. Le niveau de formation est divisé en 3 classes :

- niveau de formation élémentaire («école obligatoire » ou «apprentissage ») ;

- niveau moyen de formation («maturité/diplôme »);

- niveau élevé de formation (« haute école » ou « université »).

\subsubsection{Paramètres significatifs}

Les paramètres significatifs pour la problématique du radon sont les suivants :

- statut du logement : l'échantillon regroupe 381 propriétaires et 419 locataires ;

- risque en radon de la commune (Fig. 1) : toutes les communes de Suisse ont été classées selon leur risque en radon sur la base de la moyenne arithmétique (MA) 


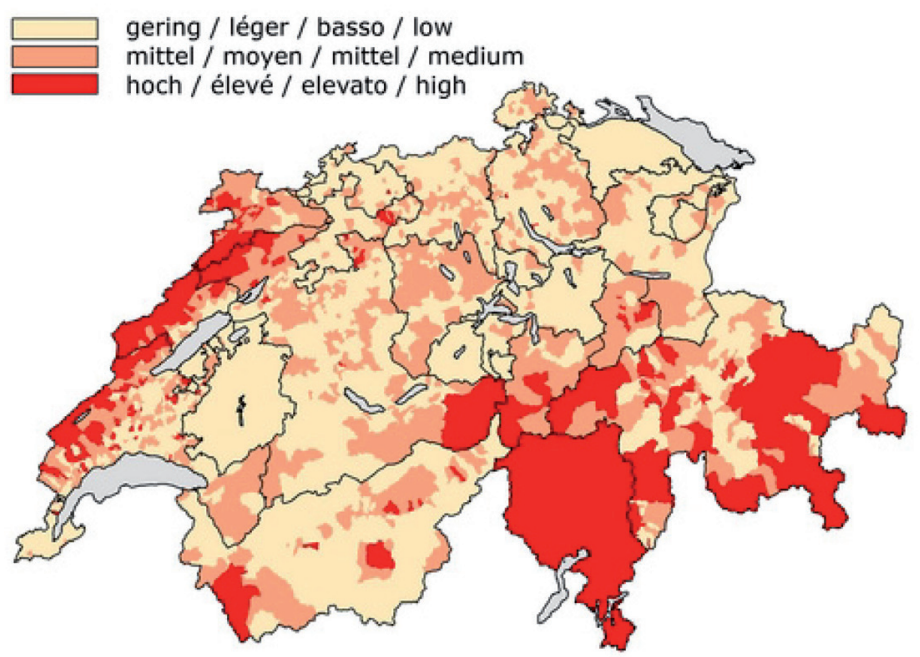

Figure 1 - Carte du radon en Suisse (2009).

Radon map in Switzerland (2009).

des mesures réalisées dans des locaux d'habitation et de séjour : risque élevé (MA supérieure à $200 \mathrm{~Bq} / \mathrm{m}^{3}$ ), risque moyen (MA entre 100 et $200 \mathrm{~Bq} / \mathrm{m}^{3}$ ), risque léger (MA inférieure à $\left.100 \mathrm{~Bq} / \mathrm{m}^{3}\right)^{3}$. L'OFSP a émis des recommandations selon le risque en $\operatorname{radon}^{4}$ et l'effort de sensibilisation du public s'est concentré principalement dans les régions à risque élevé et moyen. Ce paramètre sera donc intégré dans l'analyse de chaque question. Dans l'échantillon de 800 personnes, 53 habitent dans une commune à risque élevé, 147 dans un commune à risque moyen et 600 dans une commune à risque léger.

\subsubsection{Groupe du collectif+}

Il s'agit de faire la distinction entre les personnes qui connaissent le radon et celles qui ne le connaissent pas, ou qui en ont déjà entendu parler, mais ne savent pas que c'est un gaz radioactif. Font partie du groupe du collectif+ les personnes qui ont déjà entendu parler du radon et qui savent qu'il s'agit d'un gaz radioactif.

\section{Résultats}

L'analyse des résultats est structurée selon les quatre volets de l'enquête (caractéristiques du radon, effets du radon sur la santé, mesures à prendre pour se

\footnotetext{
3 Moteur de recherche par commune (sous www.ch-radon.ch).

4 Recommandations selon le risque en radon (sous www.ch-radon.ch).
} 
protéger et situation en Suisse), en gardant en tête les trois objectifs de l'étude, à savoir la définition des niveaux de connaissance actuels de la population suisse et sa variation en fonction du risque en radon dans la commune de résidence, ainsi que l'évaluation de l'évolution entre 1995 et 2008.

Selon le thème abordé, les données seront mises en valeur pour l'ensemble de la population suisse ou pour le groupe du collectif + , en fonction des paramètres sociodémographiques et des paramètres significatifs. Pour une meilleure lisibilité du rapport, les chiffres ont été arrondis dans les textes. Les valeurs sont toutes pondérées, y compris les bases.

\subsection{Caractéristiques du radon}

\subsubsection{Questionnaire}

Ce volet contient cinq questions. Les réponses exactes sont inscrites en italique.

1. Est-ce que vous avez déjà entendu parler du radon ? Oui / non (échantillon : 800 personnes, indicateur).

2. Qu'est-ce que le radon à votre avis ? Un gaz, un produit de lessive, une denrée alimentaire, un médicament (échantillon : 800 personnes).

3. À votre avis, est-ce que le radon est... ? Radioactif, insipide, incolore, inodore, fluorescent (échantillon: 434 personnes, question posée aux personnes ayant répondu correctement à la question 2).

4. À votre avis, où, à part la roche et le sol, est-ce que le radon se trouve également en forte concentration ? Réponses à choix: en plein air / à l'intérieur des bâtiments (échantillon : 800 personnes, indicateur).

5. À votre avis, où, à part la roche et le sol, est-ce que le radon se trouve également en forte concentration ? Réponses à choix : dans les étages supérieurs / dans la cave (échantillon : 800 personnes).

\subsubsection{Analyse}

La question « est-ce que vous avez déjà entendu parler du radon ? » est centrale pour évaluer la connaissance actuelle de la population en Suisse. Suite à l'analyse des réponses, on constate que près de $40 \%$ de la population suisse a déjà entendu parler du radon, ce qui représente une augmentation de $8 \%$ par rapport à l'enquête de 1995 , où ce taux était d'environ $32 \%$.

Certains paramètres sociodémographiques ont une influence sur les réponses :

- les hommes (43\% de oui) connaissent mieux la problématique que les femmes (36\% de oui) ; 


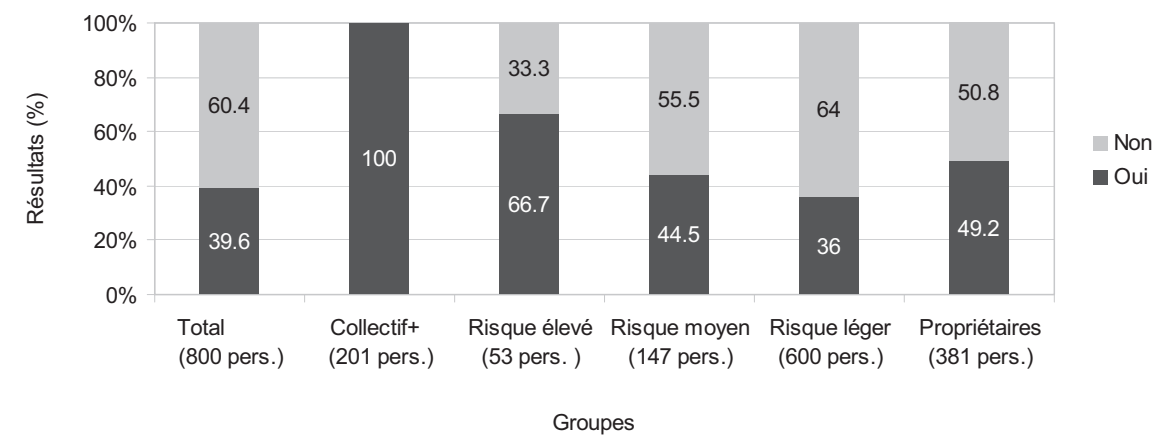

Figure 2 - «Est-ce que vous avez déjà entendu parler du radon?».

“Have you already heard of radon?".

- la classe d'âge des 18-34 ans obtient des résultats nettement inférieurs (27\% de oui) à la classe des 35-54 ans (38\% de oui) et à la classe des 55-74 ans (58\% de oui) ;

- les personnes avec un niveau de formation élémentaire connaissent moins bien la problématique (32\% de oui) que celles avec un niveau moyen de formation (40\% de oui) et que celle avec un niveau élevé de formation (55\% de oui) ;

- avec 69 \% de oui, la Suisse italienne connaît mieux le radon que la Suisse allemande (40\% de oui) et que la Suisse romande (31\% de oui).

Les paramètres jugés significatifs le sont effectivement (Fig. 2) :

- on constate que $67 \%$ des résidents des communes à risque en radon élevé en ont déjà entendu parler, contre $45 \%$ dans les communes à risque moyen et $36 \%$ dans les communes à risque léger ;

- avec $49 \%$ de oui, les propriétaires connaissent mieux la problématique du radon que les locataires ( $31 \%$ de oui).

À la question « qu'est-ce que le radon : un gaz, un produit de lessive, une denrée alimentaire ou un médicament ? », près de $54 \%$ des 800 personnes interrogées ont choisit la réponse correcte, c'est-à-dire « un gaz » (Fig. 3). À noter que $90 \%$ des personnes ayant répondu correctement à la première question ont également répondu correctement à la deuxième. Parmi les personnes qui se sont trompées, environ $42 \%$ ont répondu que le radon est un médicament. Tout comme à la première question, les paramètres significatifs ont une influence sur les résultats. Près de $76 \%$ des habitants de régions à risque élevé ont donné la bonne réponse, contre $56 \%$ dans les régions à risque moyen et $52 \%$ dans les régions à risque léger. 


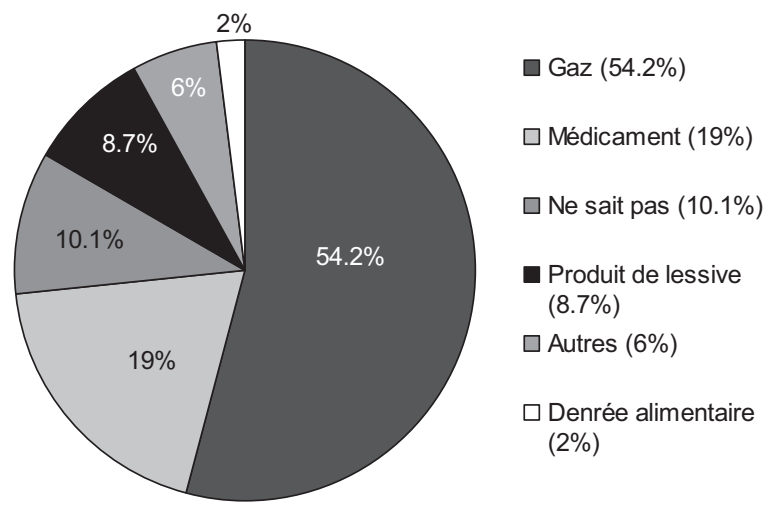

Figure 3 - «Qu'est-ce que le radon à votre avis? ».

"What do you think radon is?".

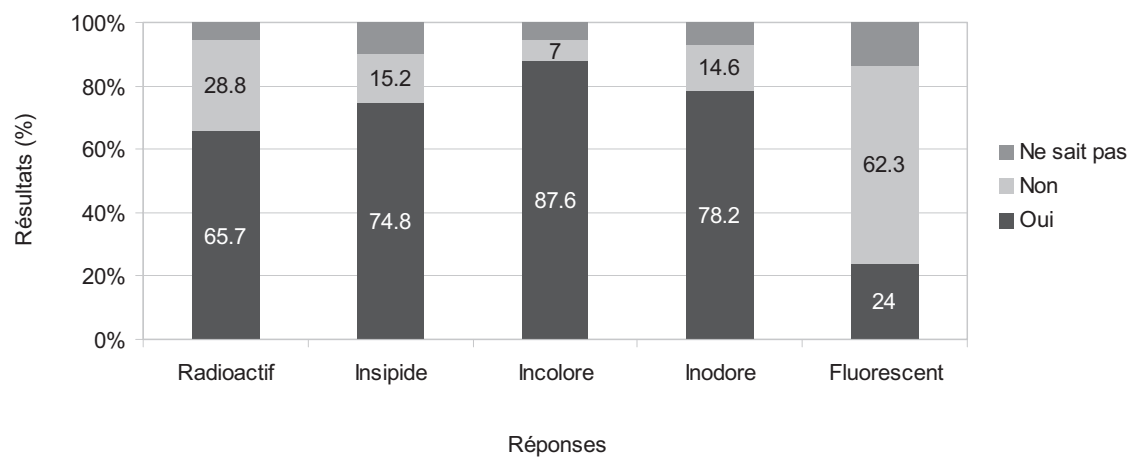

Figure 4 - «À votre avis, est-ce que le radon est... ?».

"In your opinion, radon is... ?".

La question « est-ce que le radon est : radioactif, insipide, incolore, inodore ou fluorescent ? » n'a été posée qu'aux 434 personnes ayant répondu que le radon est un gaz. L'analyse montre que près de $66 \%$ des personnes interrogées savent que le radon est radioactif (Fig. 4).

On constate ici que les paramètres significatifs n'ont aucune influence sur les réponses. En effet, le taux de réponses correctes est le même quel que soit le risque en radon dans la commune de résidence (environ $65 \%$ ). Les propriétaires et locataires obtiennent également des résultats identiques (environ $65 \%$ de réponses exactes) sur la nature radioactive du radon. 
Comme indiqué au point 2.2.3, la fraction de la population qui a déjà entendu parler du radon et qui sait qu'il s'agit d'un gaz radioactif est appelée « collectif+ » et regroupe près de 200 personnes. Ce groupe est constitué de :

- les 35-54 ans sont majoritaires (40\%), suivi des 55-74 ans (35\%) et des 18-34 $(25 \%)$;

- $73 \%$ des personnes habitent en Suisse alémanique, $19 \%$ en Suisse romande et $8 \%$ en Suisse italienne ;

- $38 \%$ des personnes du collectif+ ont un niveau élevé de formation, contre $27 \%$ sur l'ensemble de la Suisse ;

- 21 personnes résident dans une commune à risque en radon élevé, 36 dans un commune à risque moyen et 144 dans un commune à risque léger (soit $72 \%$ ) ;

- $57 \%$ de propriétaires et $43 \%$ de locataires.

La question « à part dans la roche et le sol, où le radon se trouve-t-il en forte concentration ? » a été posée aux 800 personnes interviewées. Dans le texte introductif, une définition a été fournie aux personnes qui ne savaient pas que le radon est un gaz. D'une part, il fallait choisir entre l'intérieur des bâtiments et l'air extérieur et d'autre part, entre la cave et les étages supérieurs :

- dans le premier volet de la question, environ $66 \%$ des personnes interrogées ont répondu correctement, à savoir à l'intérieur des bâtiments, ce qui représente une amélioration de plus de $10 \%$ par rapport à l'enquête de 1995 . Avec $77 \%$ de réponses exactes, le collectif+ dépasse d'environ $10 \%$ l'ensemble de la population. Dans ce groupe, pratiquement tous les habitants des régions à risque élevé, soit $95 \%$, ont répondu correctement à cette question ;

- dans le deuxième volet de la question, plus de $81 \%$ des 800 personnes interrogées ont choisies la réponse exacte, c'est-à-dire dans la cave. Le collectif+ a fourni la bonne réponse dans $85 \%$ des cas, ce qui est légèrement supérieur à l'ensemble de la population.

\subsection{Effets du radon sur la santé}

\subsubsection{Questionnaire}

Ce volet contient trois questions. Les réponses exactes sont inscrites en italique.

6. Pensez-vous que la présence de radon dans les habitations peut nuire à votre santé ? Oui-non (échantillon : 800 personnes, indicateur).

7. À votre avis, le radon est à l'origine de ? Migraine, diarrhée, cancer $d u$ poumon, affection dermatologique, troubles cardiaques (échantillon : 677 personnes ayant répondu oui à la question 6). 


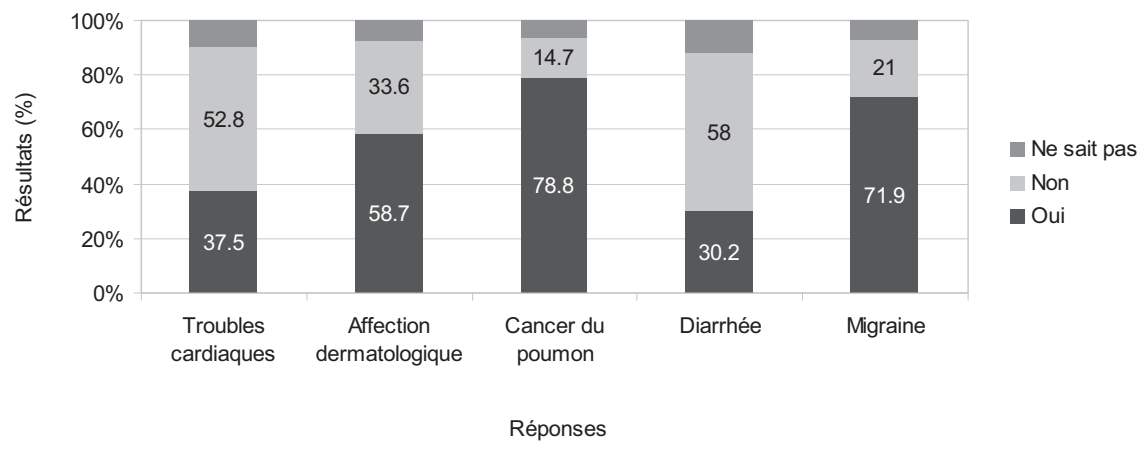

Figure 5 - « À votre avis, quels sont les effets du radon sur la santé ? ».

"In your opinion, what are the effects of radon on health?".

8. À votre avis, quelles sont les personnes les plus touchées par le cancer du poumon? Les enfants, les femmes enceintes, les personnes âgées, toute la population (échantillon : 800 personnes).

\subsubsection{Analyse}

Près de $85 \%$ des 800 personnes interrogées sont conscientes que le radon est nocif pour la santé. Cela représente une augmentation de plus de $13 \%$ depuis l'enquête de 1995. Quant au collectift, $91 \%$ des personnes interrogées sont conscientes que le radon est nocif pour la santé. Pour les personnes de ce groupe vivant dans les régions à risque élevé, ce taux atteint $98 \%$.

La question des effets du radon sur la santé a été uniquement posée aux 677 personnes ayant répondu que le radon est nocif pour la santé. La question est de type multichotomique à choix multiples, c'est-à-dire que plusieurs réponses exactes sont possibles. Dans la réalité, le seul effet connu du radon sur la santé est le cancer du poumon. Près de $79 \%$ des personnes interrogées ont répondu correctement (Fig. 5). À noter que la majorité des interviewés pensent que le radon provoque aussi d'autres effets sur la santé, notamment la migraine à $72 \%$ et les affections dermatologiques à $59 \%$. Environ $28 \%$ des personnes sondées en Suisse alémanique et $38 \%$ en Suisse romande pensent que le radon peut être à l'origine de diarrhée, alors que ce taux est nettement plus bas, soit $20 \%$, dans la région italophone.

Dans le collectif + , près de $80 \%$ des individus interrogés pensent que le radon provoque le cancer du poumon, ce qui est un résultat similaire à l'ensemble de la Suisse. Par contre, ce groupe se trompe légèrement moins que la totalité de la 
population sur les autres effets du radon sur la santé, même si les mêmes tendances sont observées. Par exemple, le groupe a répondu à $62 \%$ que le radon induit également des migraines et à $54 \%$ des affections dermatologiques.

À la question « quelles sont les personnes les plus touchées par le cancer du poumon ? », presque $60 \%$ des 800 personnes sondées ont répondu de manière exacte, à savoir « toute la population ».

\subsection{Mesures à prendre pour se protéger}

\subsubsection{Questionnaire}

Ce volet contient six questions. Les réponses exactes sont inscrites en italique :

9. Pensez-vous qu'il soit possible de mesurer la concentration en radon dans les bâtiments ? Oui-non (échantillon : 800 personnes).

10. Avez-vous déjà fait mesurer la concentration en radon dans votre maison ? Oui-non (échantillon : 736 personnes qui savent que le radon peut être mesuré).

11. Est-ce que vous prévoyez de faire mesurer la concentration en radon dans votre maison ? Oui définitivement, oui probablement, probablement pas, définitivement pas (échantillon : 698 pers. qui savent que le radon peut être mesuré, mais ne l'ont pas encore fait, échantillon : 64 pers. qui ne savent pas que le radon peut être mesuré).

12. Est-ce que vous pensez qu'en cas de forte concentration de radon dans un bâtiment on peut entreprendre quelque chose pour la réduire ? Oui-non (échantillon : 800 personnes, indicateur).

13. À votre avis, la mise en œuvre des méthodes pour empêcher l'entrée du radon dans les appartements est... très compliquée, assez compliquée, assez simple, très simple ? (échantillon: 800 personnes).

14. En ce qui concerne les coûts, sont-ils... très élevés, moyennement élevés, peu élevés ? (échantillon : 800 personnes).

\subsubsection{Analyse}

Lorsqu'on demande aux personnes interrogées si elles pensent qu'il est possible de mesurer la concentration en radon dans les bâtiments, $92 \%$ de la population suisse répond par l'affirmative (dont 92 des 93 personnes résidant dans une zone à risque élevé). Dans le collectif+, ce taux atteint $97 \%$, et même $100 \%$ dans les régions à risque élevé (Fig. 6). 


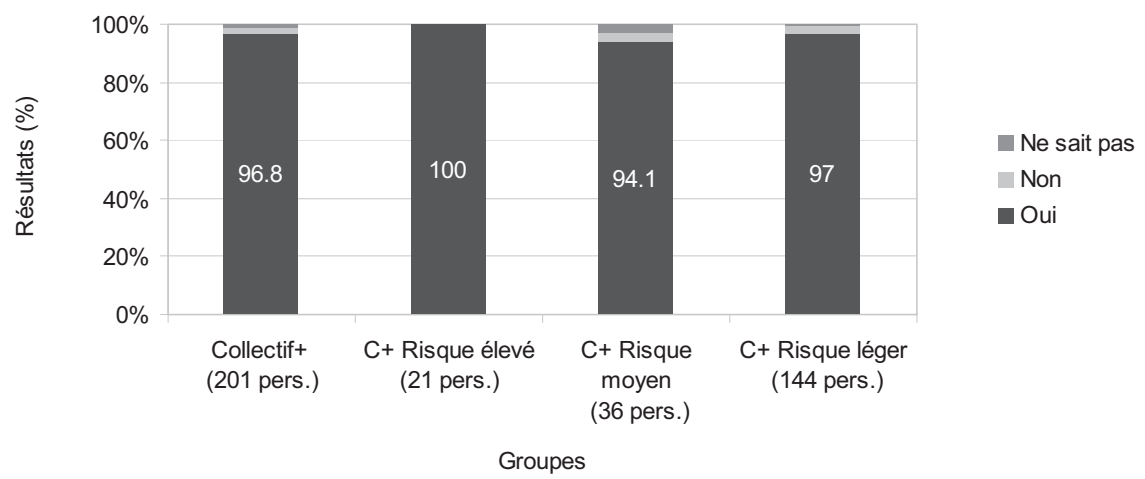

Figure 6 - Collectif+ : «Pensez-vous qu'il soit possible de mesurer la concentration en radon dans les bâtiments? ».

Collective+: "Do you think that it is possible to measure radon concentration in building?".

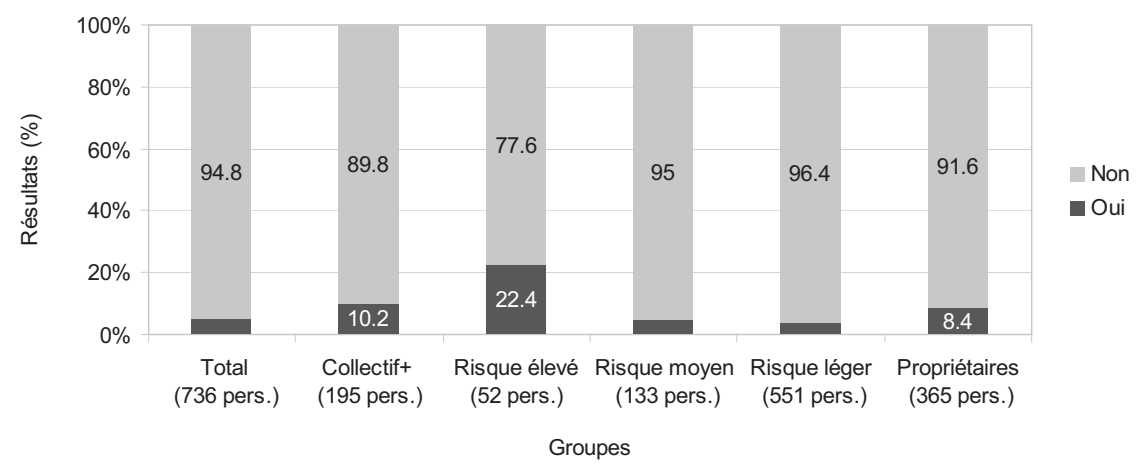

Figure 7 - « Avez-vous déjà fait mesurer la concentration en radon dans votre maison ? ».

“Have you already measured the radon concentration in your house?”.

La question « avez-vous déjà fait mesurer la concentration en radon dans votre maison ? » a été posée aux 736 personnes qui pensaient que le radon peut être mesuré (Fig. 7). On constate que seules $5 \%$ des personnes sondées en Suisse ont déjà procédé à la mesure. Plus de $8 \%$ des propriétaires ont déjà fait mesurer le radon chez eux, contre $2 \%$ de locataires. À noter toutefois que la base des « oui » n'est que de 8 personnes pour les locataires. Seuls $4 \%$ des résidents des régions à risque léger ont répondu par l'affirmative, alors qu'ils sont $22 \%$ dans les régions à risque élevé. 
Dans le collectif+, $10 \%$ des personnes interrogées ont déjà fait procéder à une mesure du radon. Dans ce groupe, près de $30 \%$ des personnes qui ont fait une mesure habite dans une commune à risque élevé, contre $13 \%$ dans une commune à risque moyen et $6 \%$ dans une commune à risque léger.

On a ensuite demandé au $95 \%$ des interrogés qui n’ont jamais fait mesurer le radon chez eux, s'ils comptaient le faire, en les divisant en deux groupes :

- après une brève explication sur la mesure du radon, on a posé la question aux 64 personnes, qui n'étaient pas au courant de cette possibilité. Seules $34 \%$ ont répondu favorablement, mais la majorité des personnes interrogées habitent en région à risque léger et sont donc peu concernées par la problématique du radon ;

- environ $26 \%$ des 698 individus qui savent que le radon peut être mesuré sans l'avoir fait eux-mêmes, sont favorables, ce taux atteignant presque $50 \%$ dans les régions avec un risque en radon élevé, c'est-à-dire le double des deux autres régions.

Lors de l'enquête de 1995, 64 \% de la population était d'avis que les solutions permettaient de réduire la concentration en radon dans un bâtiment. En 2008, ce taux est de $76 \%$, soit $12 \%$ de plus qu'en 1995. Les réponses du collectif+ sont légèrement meilleures, surtout dans les zones à risque élevé (93\% de réponses exactes).

À la question « à votre avis, la mise en œuvre des méthodes pour empêcher l'entrée du radon dans les appartements est ? Très compliquée, assez compliquée, assez simple, très simple ? », les opinions sont très partagées, mais la majorité des Suisses penche plutôt pour la réponse « simple » à $64 \%$. Les personnes avec un niveau de formation élémentaire ont tendance à trouver les méthodes de construction plus compliquées que celles avec un niveau de formation moyen et élevé. En Suisse alémanique, $17 \%$ des interrogés pensent que ces méthodes sont « très simples », contre $10 \%$ en Suisse romande et $8 \%$ en Suisse italienne. Les résultats sont du même ordre de grandeur dans le collectif+.

En ce qui concerne la question des coûts des méthodes de construction préventives et d'assainissement (très élevés, moyennement élevés ou peu élevés), $29 \%$ des Suisses trouvent les coûts très élevés, $51 \%$ moyennement élevés et $16 \%$ peu élevés (4\% d'abstention). Le taux des personnes étant d'avis que ces méthodes sont bon marché est plus élevé dans la région italophone (28\%) que dans le reste de la Suisse, soit $17 \%$ en Suisse romande et $14 \%$ en Suisse alémanique. Cette tendance se répète avec les individus des régions à risque élevé, dont $25 \%$ pensent que les coûts sont peu élevés, contre environ $15 \%$ dans les régions à risque moyen et léger. 


\subsection{Situation en Suisse}

\subsubsection{Questionnaire}

Ce dernier volet contient sept questions. Les réponses exactes sont inscrites en italique :

15. L'Office fédéral de la santé publique est chargé de l'information du public sur le radon par des actions médiatiques et par de la documentation. En avez-vous eu connaissance? Oui-non (échantillon : 800 personnes).

16. Est-ce que vous avez eu connaissance de cette information sur le radon par le biais de ? Brochure, site internet, article dans les journaux, séances d'information, stands d'information (échantillon : 178 personnes qui connaissent les actions médiatiques de l'OFSP).

17. Savez-vous que votre canton a organisé des campagnes de mesures du radon dans les habitations ? Oui-non (échantillon : 800 personnes).

18. Savez-vous si le risque en radon dans votre commune est... élevé, moyen ou léger? (échantillon : 800 personnes).

19. Selon vous, les régions suivantes sont-elles particulièrement problématiques du point de vue du radon ? Tessin, Grisons, Arc lémanique, Arc jurassien, Plateau suisse (échantillon : 800 personnes).

20. À votre avis est-ce qu'un locataire habitant une maison dépassant la limite légale peut exiger un assainissement par le propriétaire ? Oui-non (échantillon : 800 personnes).

21. D'après vous, qui doit, selon l'ordonnance, prendre en charge les frais d'assainissement d'un bâtiment ? Les autorités, les propriétaires, les locataires, les assurances (échantillon: 800 personnes).

\subsubsection{Analyse}

Ce dernier volet du questionnaire débute par la question suivante : «l'Office fédéral de la santé publique est chargé de l'information du public sur le radon par des actions médiatiques et par de la documentation. En avez-vous eu connaissance ? ». On observe (Fig. 8) que $22 \%$ de la population suisse a déjà eu connaissance de ces informations, ce taux atteignant $37 \%$ dans les zones à risque en radon élevé (contre $19 \%$ dans les régions à risque léger). Les propriétaires sont aussi plus au courant de l'information diffusée, car ils obtiennent des résultats significativement plus élevés (28\%) que les locataires (17\%). Le groupe le mieux informé reste le collectif + , car $40 \%$ des personnes interrogées répondent avoir eu connaissance des informations diffusées. 


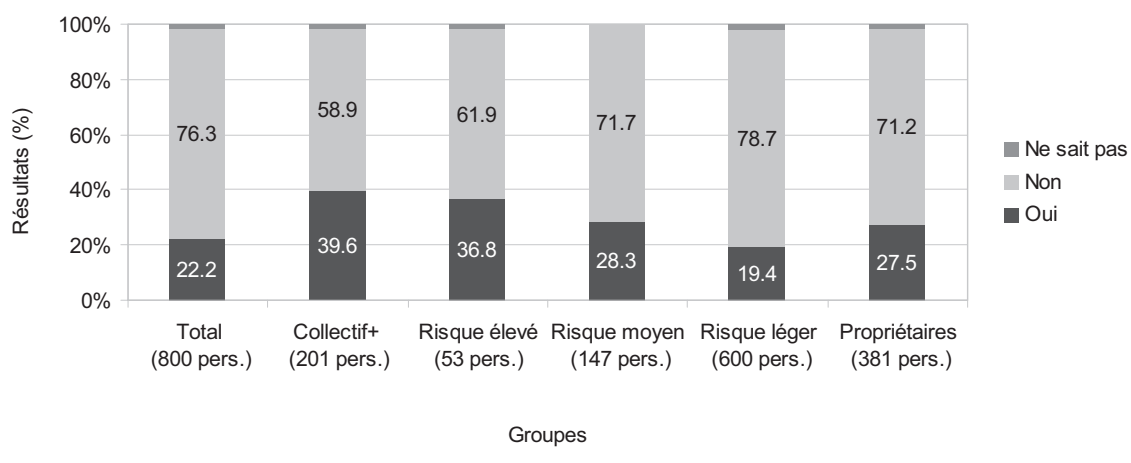

Figure 8 - «L'Office fédéral de la santé publique est chargé de l'information du public sur le radon par des actions médiatiques et par de la documentation. En avez-vous eu connaissance? ?.

"The Federal Office of Public Health (FOPH) is in charge of informing the public on radon by media actions and documentation. Were you informed about this fact?'”

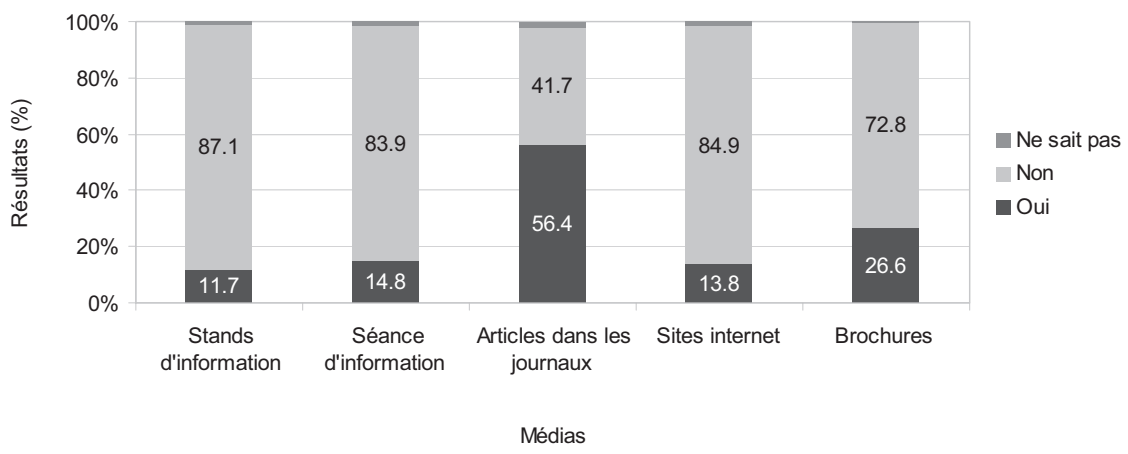

Figure 9 - "Par quel biais avez-vous eu connaissance des informations de l'OFSP ? ».

"How did you get the information from the FOPH?".

On a ensuite demandé par quel bais les 178 personnes avaient eu connaissance des informations de l'OFSP : brochure, site internet, article dans les journaux, séances d'information, stands d'information. La majorité des personnes a reçu l'information par le biais de la presse (56\%) et des brochures $(27 \%)$ et beaucoup moins par le biais d'internet ainsi que de soirées et/ou stands d'information (Fig. 9). Les résultats du collectif+ ne sont que légèrement supérieurs par rapport à l'ensemble de la Suisse.

La question "savez-vous que votre canton a organisé des campagnes de mesure du radon dans les habitations ? » a été posée aux 800 personnes de l'échantillon. Environ $11 \%$ de la population suisse a répondu par l'affirmative 


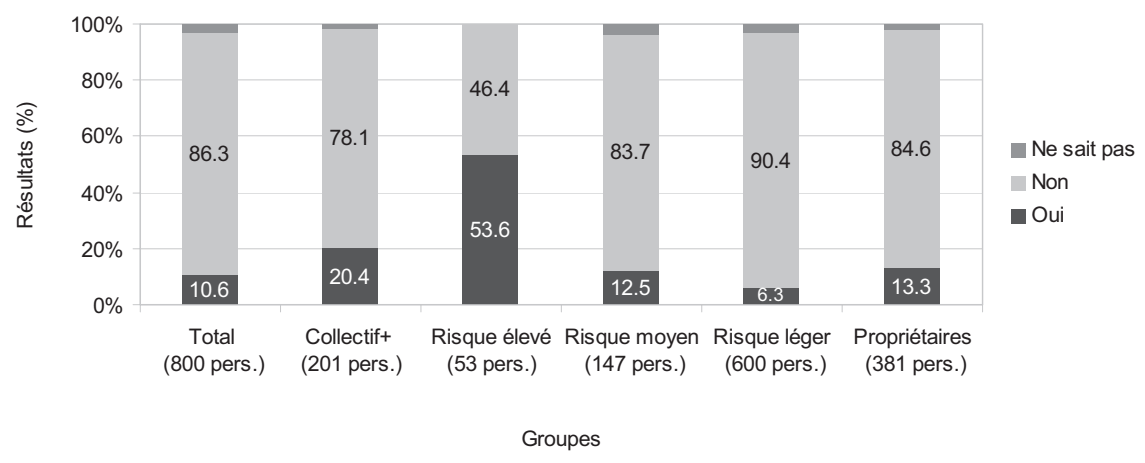

Figure 10 - «Savez-vous que votre canton a organisé des campagnes de mesures du radon dans les habitations?».

"Do you know that your canton has been organizing radon measurements campaigns in dwellings?".

(Fig. 10). On remarque sur le graphique que les personnes résidant dans les régions à risque en radon élevé ont des connaissances nettement meilleures $(54 \%)$ que les autres régions (risque moyen $12 \%$ et risque léger $6 \%$ ). C'est surtout le groupe le plus âgé qui est au courant des campagnes de mesure du radon avec un taux de « oui » de $15 \%$ par rapport au 18-34 ans qui ont répondu « oui » à $6 \%$ seulement. La région italophone est la mieux informée avec des résultats atteignant $62 \%$ par rapport à la Suisse romande $(11 \%)$ et la Suisse alémanique $(7 \%)$.

Dans le collectif + , environ $20 \%$ des personnes interrogées étaient au courant des campagnes de mesures. Dans ce groupe, près de $83 \%$ des individus domiciliés dans une région à risque élevé ont eu connaissance des campagnes de mesures, ce qui est un résultat clairement supérieur aux individus des régions à risque léger $(13 \%)$ et moyen $(15 \%)$.

On a ensuite demandé aux 800 personnes de l'échantillon si elles connaissaient le risque en radon dans leur commune de domicile, à savoir léger, moyen ou élevé. La figure 11 montre que la réponse la plus fréquente est « ne sait pas », à $44 \%$. Seules $25 \%$ des personnes interrogées (et $33 \%$ dans le groupe du collectif+) indiquent la classification correcte du risque en radon dans leur commune de domicile. Paradoxalement, on remarque que seuls $12 \%$ des habitants des régions à risque en radon élevé sont correctement informés (19\% dans le collectif+), contre $20 \%$ dans les zones à risque moyen et $27 \%$ dans les zones à risque léger. Dans les régions les plus concernées, le risque lié au radon est donc largement sous-estimé. 


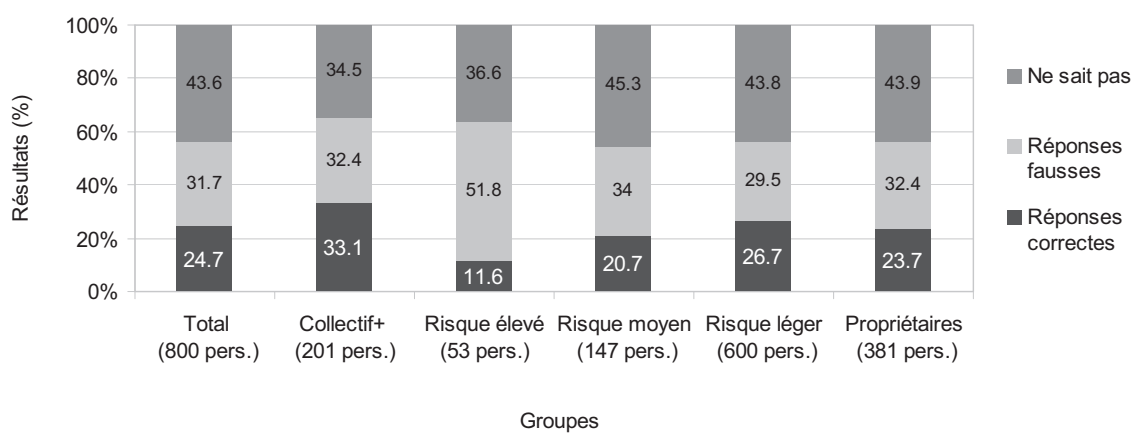

Figure 11 - «Savez-vous si le risque en radon dans votre commune est...élevé, moyen ou léger ? ».

"Do you know if the radon risk in your area... is hight, medium, low?".

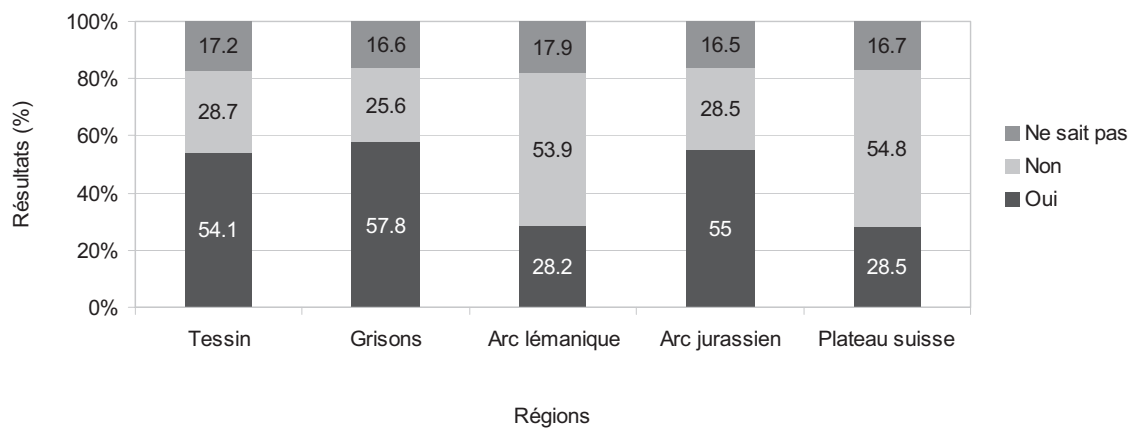

Figure 12 - «Selon vous, les régions suivantes sont-elles particulièrement problématiques du point de vue du radon?".

"According to you, are the following regions particularly problematic from the point of view of radon?".

À la question « selon vous, les régions suivantes sont-elles particulièrement problématiques du point de vue du radon?» on obtient plus de $65 \%$ de réponses correctes en Suisse (en éliminant les réponses « ne sait pas »), à savoir, « oui » pour le Tessin, les Grisons et l'Arc jurassien et « non » pour l'Arc lémanique et le Plateau suisse (Fig. 12). Les personnes habitant dans la région citée donnent généralement la bonne réponse. Par exemple pour le Tessin, $66 \%$ des tessinois jugent leur canton problématique, contre $46 \%$ des personnes interrogées en Suisse romande. Les habitants des régions francophones et italophones répondent qu'ils ne savent pas à $35 \%$ environ, contre environ $10 \%$ dans la région germanique (exception faite des réponses des italophones pour le Tessin). 
Le collectif+ fournit presque $80 \%$ de réponses correctes, soit $15 \%$ de plus que dans l'ensemble de la Suisse (en éliminant les réponses « ne sait pas »).

Les deux dernières questions portent sur les aspects juridiques. En introduction, les personnes interrogées ont été informées que l'ORaP fixe une limite légale de 1000 becquerels par mètre cube $\left(\mathrm{Bq} / \mathrm{m}^{3}\right)$ à partir de laquelle un assainissement est obligatoire.

On a demandé aux personnes interrogées si, à leur avis, un locataire habitant dans une maison dépassant la limite légale pouvait exiger un assainissement par le propriétaire. Environ $80 \%$ des personnes interrogées en Suisse ont répondu correctement. Les personnes des régions à risque élevé (92\%) connaissent mieux le droit des locataires que celles des régions à risque léger (77\%). Les résultats du collectif+ sont légèrement meilleurs ( $84 \%$ ) que ceux de l'ensemble de la Suisse, surtout dans les régions à risque moyen et élevé (90\% de réponses correctes).

La dernière question de l'enquête est « d'après vous, qui doit, selon l'ordonnance, prendre en charge les frais d'assainissement d'un bâtiment ? Les autorités, les propriétaires de bâtiment, les locataires, les assurances ? ». Près de $68 \%$ des personnes interrogées donnent la bonne réponse, à savoir «les propriétaires de bâtiment ».

\section{Conclusions}

Grâce à cette enquête, on a pu observer l'évolution de la connaissance sur le radon en Suisse depuis 1995 et appréhender la perception actuelle du public, en faisant ressortir des différences régionales.

Par le biais des quatre indicateurs, on constate que la connaissance de la population suisse a augmenté d'environ $10 \%$ entre 1995 et 2008 :

- oui, j'ai déjà entendu parler du radon : 1995 : $32 \%, 2008$ : $40 \%$;

- le radon est un problème à l'intérieur et non à l'extérieur des bâtiments : 1995 : $55 \%, 2008: 66 \%$;

- le radon est nocif pour la santé : 1995 : $72 \%, 2008: 85 \%$;

- des solutions permettent de réduire la concentration en radon dans un bâtiment ; 1995 : $64 \%, 2008$ : $76 \%$.

Les résultats de l'enquête de 2008 peuvent se résumer ainsi :

- pour aborder le niveau de connaissance actuel de la population suisse en matière de radon, il est indispensable de tenir compte de la région de domicile des personnes sondées. On constate en effet que les connaissances des habitants de communes à risque élevé dépassent très nettement le reste de la 
Suisse. Par exemple, environ $40 \%$ de la population suisse indique avoir déjà entendu parler du radon, contre $67 \%$ dans les régions à risque élevé. Par contre, les différences sont peu marquées entre les réponses des communes à risque moyen et léger ;

- à l'intérieur de l'enquête, un collectif a été formé comprenant les personnes les mieux informées sur la problématique du radon (celles qui savent qu'il s'agit d'un gaz radioactif ; 200 personnes). Ce groupe correspond à la structure de la population suisse aux niveaux du genre et de la région linguistique, mais il comprend plus de personnes âgées, ainsi que d'individus ayant un niveau élevé de formation et propriétaire immobilier. En proportion, les personnes de ce groupe donnent systématiquement de meilleures réponses que l'ensemble de la Suisse ;

- environ $80 \%$ de la population suisse est consciente que le radon est nocif pour la santé et sait qu'il peut provoquer le cancer du poumon. Toutefois, la majorité des interviewés pensent que le radon induit aussi d'autres effets sur la santé, notamment la migraine et les affections dermatologiques ;

- près de $92 \%$ de la population suisse pensent qu'il est possible de mesurer le radon, ce taux atteignant presque $100 \%$ dans les régions à risque élevé. Effet direct des campagnes de mesures systématiques organisées en Suisse, près de la moitié des personnes résidant dans des communes à risque élevé connaissent cette possibilité et plus de $20 \%$ ont déjà fait réaliser une mesure (contre $5 \%$ seulement en Suisse);

- la population a généralement une bonne idée de la localisation des régions problématiques, à savoir le Tessin, les Grisons et l'Arc jurassien. Paradoxalement, lorsqu' on demande aux interviewés le risque en radon dans leur commune de domicile, le risque est largement sous-estimé dans les régions les plus concernées. Le citoyen suisse a donc tendance à considérer que le radon est un problème chez les autres, mais pas chez lui ;

- la plupart des personnes interrogées en Suisse sont au courant des droits et des devoirs des propriétaires et des locataires fixés dans l'ORaP. Globalement, les propriétaires connaissent légèrement mieux la problématique que des locataires, mais ces différences sont peu marquées.

Dans la situation actuelle, le thème du radon est bien connu dans les communes à risque élevé, ce qui démontre que la communication ciblée accompagnant les campagnes de mesures a porté ses fruits et doit être poursuivie. Le bilan est moins positif pour les communes à risque moyen et léger, où bon nombre d'individus n'ont jamais entendu parler du radon, alors que certains types de bâtiment sont potentiellement à risque dans toute la Suisse (ancienne construction, sous-sol habité, cave naturelle ou bâtiment sans sous-sol). 
Il serait souhaitable qu'un large public en Suisse acquière des notions de base sur le radon. Les nouvelles directives de l'Organisation mondiale de la santé (OMS), avec des niveaux de référence plus bas, contribueront certainement à consolider le programme du radon en Suisse et à renfoncer la perception de la population sur ce sujet (WHO, 2009).

\section{RÉFÉRENCES}

IPSO (1995) Bekanntheit von Radon in der Bevölkerung, Dübendorf.

Kay D.A., Cliffs A.P. (2004) Statistics, John Wiley and Sons.

OFS (2006) Niveaux géographiques de la Suisse, Recensement fédéral de 2000.

OFS (2007) Encyclopédie statistique de la Suisse, Population résidante permanente selon l'âge et le genre, Document no. 20563.

ORaP (1994) Ordonnance sur la radioprotection (ORaP, RS 814.501) du 22 juin 1994.

WHO (2009) World Health Organization, WHO-Handbook on indoor radon, a public health perspective. 